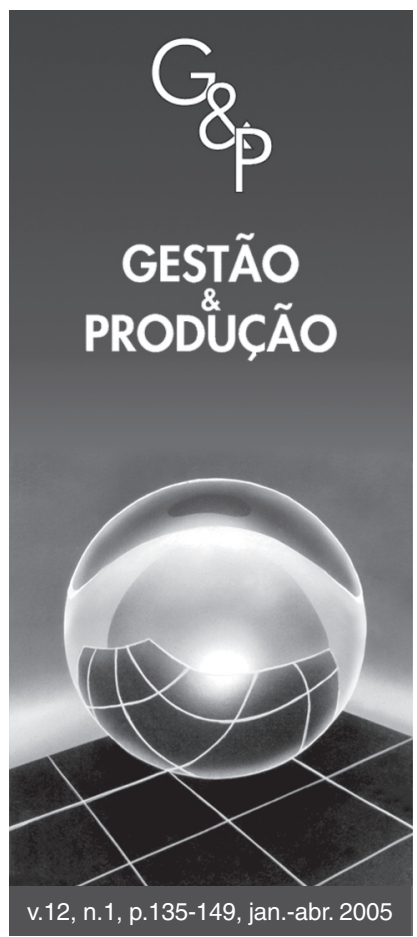

\title{
OTIMIZAÇÃO NA PROGRAMAÇÃO DA PRODUÇÃO DE GRÃOS ELETROFUNDIDOS: UM ESTUDO DE CASO
}

\author{
José Roberto Dale Luche \\ Reinaldo Morabito \\ Departamento de Engenharia de Produção, \\ Universidade Federal de São Carlos, SP, \\ CEP 13565-905, São Carlos, SP, \\ e-mail: dluche@gmail.com; morabito@ power.ufscar.br
}

Resumo

Este trabalho apresenta modelos de otimização para apoiar decisões do Planejamento e Controle da Produção $(P C P)$ na indústria de grãos eletrofundidos. Um estudo de caso foi realizado numa empresa no Estado de São Paulo com o objetivo de contribuir para aumentar a produtividade e melhorar o nível de serviço aos clientes no atendimento dos prazos de entrega. Para isso, foram aplicados modelos de programação linear inteira mista para auxiliar particularmente nas decisões da programação da produção e analisar os resultados obtidos. Os modelos combinam modelos conhecidos de seleção de processos e dimensionamento de lotes monoestágio. Otimizar tal programação na indústria de grãos eletrofundidos não é uma tarefa simples, principalmente devido à grandeza dos tempos de preparação dos equipamentos, à diversidade de produtos e às limitações dos prazos de entrega da carteira de pedidos. Os resultados mostram que os modelos são capazes de gerar soluções melhores do que as utilizadas pela empresa.

Palavras-chave: programação da produção, dimensionamento de lotes, grãos eletrofundidos.

\section{Introdução}

O objeto de pesquisa deste trabalho é o Planejamento e Controle da Produção (PCP) na indústria de grãos eletrofundidos, que possui diversas unidades de produção na região sudeste do país, em particular, no Estado de São Paulo. O trabalho é baseado num estudo de caso em uma empresa localizada na cidade de Salto, SP, que emprega aproximadamente 150 funcionários e tem uma produção mensal de mais de duas mil toneladas de matérias-primas granulares eletrofundidas. Esta empresa consome grandes quantidades de energia elétrica na produção de grãos eletrofundidos, alcançando uma média mensal de $14 \mathrm{MW} / \mathrm{h}$, o que a colocou numa situação difícil com a recente crise energética no país e foi fonte de estímulo na pesquisa de alternativas para otimizar o processo e o planejamento da produção. Diversas decisões importantes são consideradas no PCP da fábrica, em particular, com respeito aos níveis de estoques de matérias-primas e produtos intermediários, e à programação da produção dos fornos e processos subseqüentes, como britagem, moagem e classificação, em função da demanda de produtos finais. Estas decisões são típicas em empresas deste setor.

Neste trabalho são aplicados modelos de programação linear inteira mista para auxiliar particularmente nas decisões da programação da produção e analisar os resultados obtidos. Tais modelos combinam modelos conhecidos de seleção de processos e dimensionamento de lotes monoestágio, e podem ser vistos como modelos de dimensionamento de lotes que, em vez de "lotes de produtos", utilizam "lotes de processos" para produzir um conjunto de produtos. Otimizar tal programação na indústria de grãos eletrofundidos não é uma tarefa simples, principalmente devido à grandeza dos tempos de preparação dos equipamentos, à diversidade de produtos e às limitações 
dos prazos de entrega da carteira de pedidos. Gerentes de produção em empresas deste setor, em geral, encontram dificuldades para programar a produção devido, entre outros, à natureza combinatória do problema. Técnicas de programação matemática, que podem trazer grandes benefícios para o processo de tomada de decisões no PCP destas empresas, parecem não ter sido até agora efetivamente aplicadas para tratar tal problema. Conforme visto neste estudo de caso, é comum um programa de produção precisar ser modificado várias vezes devido a imprevistos ou pedidos urgentes, o que reforça a importância de um modelo capaz de gerar em tempo razoável, programas de produção eficientes.

Este artigo está organizado da seguinte maneira: na próxima seção é brevemente apresentada a indústria de grãos eletrofundidos. A discussão é baseada na planta da empresa estudada, mas também se aplica a outras empresas do setor. Na seção 3 são combinados conceitos de modelos de seleção de processos e dimensionamento de lotes para o problema do estudo de caso. O primeiro modelo proposto procura minimizar o número de períodos (MNP) necessários para produção dos itens demandados. O segundo modelo (MFP - Minimizar Falta de Produção) procura minimizar a quantidade de produtos demandados não produzidos, ou seja, consiste em minimizar a falta de produção e os atrasos na entrega. Na seção 4 são apresentados os resultados computacionais obtidos resolvendo-se os dois modelos MNP e MFP na linguagem de modelagem GAMS (com o solver CPLEX), aplicados a dados simulados e dados reais da empresa. Os resultados mostram que os modelos são consistentes e capazes de gerar soluções melhores do que a empresa. Finalmente, na seção 5 , apresentamos as conclusões e perspectivas para pesquisa futura.

\section{Definição do problema}

\subsection{A indústria de grãos eletrofundidos}

A planta estudada está dividida em duas fábricas. A primeira produz carbeto de silício (SiC), um mineral sintético também conhecido por carbureto de silício. Apresenta características físico-químicas de alta dureza, alta refratariedade e alta resistência química, qualificando-o para aplicações que exigem alto nível de desempenho. O carbeto de silício perde em dureza apenas para o diamante, que é o único material capaz de riscá-lo, mas ele tem pouca resistência a impactos devido a sua baixa ductilidade. Como exemplo da aplicação do carbeto de silício podemos citar: materiais refratários aplicados na indústria siderúrgica e materiais abrasivos (lixas e rebolos), os quais são utilizados no acabamento superficial de mármores, granitos, vidros e outros materiais. $\mathrm{O}$ carbeto de silício pode ainda ser aplicado na indústria metalúrgica dentro de composições metálicas ferrosas como agente desoxidante e carburante como, por exemplo, na produção de aço e de ferro fundido (Alcoa, 2002).

A segunda fábrica produz óxido de alumínio (AlO) e está dividida em duas seções, a primeira produz óxido de alumínio branco e a segunda produz óxido de alumínio marrom. Todos os produtos apresentam-se na forma de grãos cujos tamanhos variam desde alguns centímetros até poucos micrômetros.

\subsection{Descrição dos processos}

$\mathrm{Na}$ fábrica de $\mathrm{SiC}$, o quartzo e o coque de petróleo são utilizados como as principais matérias-primas. Elas são transportadas até um misturador onde são adicionados outros elementos para atingir a composição ideal do $\mathrm{SiC}$. A mistura segue para fornos (fornos Acheson) com paredes contendo eletrodos de grafite ligados a um gerador elétrico através de barramentos de cobre. Alma de pó de grafite é colocada no meio da mistura para conduzir a eletricidade entre os eletrodos e provocar o aquecimento e transformação da matéria-prima. O material próximo à grafite é o de melhor qualidade por ser onde se formam os melhores cristais. À medida que se afasta do fio de grafite, os cristais são menores e tidos como de baixa qualidade, o que obriga os operários a separarem manualmente a parte de boa qualidade com o uso de um martelo pneumático. Em seguida o material passa por uma etapa de britagem e moagem, e posteriormente é feita uma separação magnética, em que os grãos com alto teor de ferro são separados para reprocesso. Finalmente, a última etapa desse processo de transformação classifica os grãos por meio de um conjunto de peneiras vibratórias retangulares que definem a faixa granulométrica do produto.

As etapas citadas acima podem ser vistas no diagrama de fluxo de material ilustrado na Figura 1. Mais informa-

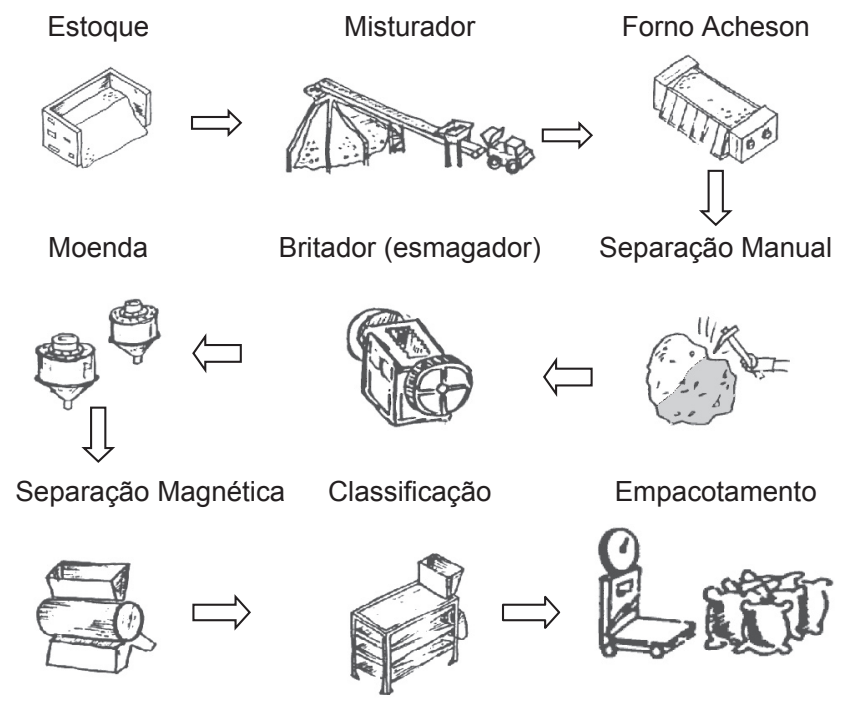

Figura 1. Diagrama de fluxo de material na fábrica de $\mathrm{SiC}$. 
ções sobre o tamanho de partículas (grãos) e regulagem dos moinhos podem ser encontradas em Allen (1990).

Com um processo de produção muito semelhante ao $\mathrm{SiC}$, na fábrica de $\mathrm{AlO}$ é feita a fusão da alumina com bauxita por um processo de redução em fase líquida em fornos (fornos Higgins). Cada forno possui três eletrodos que ficam entre a matéria-prima, aquecendo-a a uma temperatura de aproximadamente $2500{ }^{\circ} \mathrm{C}$. Após o processo de redução, o material fica descansando até alcançar a propriedade sólida, depois disso é carregado para uma área de resfriamento na qual ficará resfriando por 30 horas. O transporte nesta fábrica é realizado por uma ponte rolante, para a primeira quebra do material com rompedor hidráulico, depois disso, o material passa pelo processo de britagem e classificação.

\subsection{Foco do problema}

O sistema de produção da empresa é intermitente e repetitivo: sempre ocorrem mudanças no tipo de produto fabricado, gerando grande variedade de produtos. O layout é definido por produto - as máquinas são arranjadas de acordo com a sequiência de operações pelas quais o produto passa. Como todos os produtos possuem a mesma sequiência de operações nas diversas máquinas, o padrão de fluxo no sistema de produção desta empresa é basicamente um flow-shop. Diversas decisões importantes estão envolvidas no PCP das duas fábricas mencionadas acima, em particular, com respeito aos níveis de estoques de matérias-primas e produtos intermediários, e à programação da produção dos fornos e processos subsequientes como britagem, moagem e classificação, tudo em função da demanda de produtos finais. Conforme mencionado, otimizar a programação da produção nestas fábricas não é uma tarefa simples, principalmente devido à grandeza dos tempos de preparação dos equipamentos, à diversidade de produtos e às limitações dos prazos de entrega da carteira de pedidos.

A programação da classificação dos grãos deve ser feita em conjunto com a programação dos fornos, das britadeiras e das moendas, e envolve certas dificuldades. A classificação é feita por um conjunto de peneiras vibratórias. O conjunto tem a finalidade de separar os grãos por tamanho (o tamanho é definido pela quantidade de furos por polegada na peneira) e pode ser montado com diferentes combinações de peneiras. Existem peneiras de várias medidas diferentes. Por exemplo, o produto chamado EC31_120 é um grão que passou inicialmente por uma peneira de 100 furos por polegada e parou numa peneira de 120 furos por polegada (a quantidade de furos por polegada pode variar de 5 furos na peneira de grãos mais grossos, até 220 na peneira de grãos mais finos).

Apenas para ilustrar, a Tabela 1 apresenta as quantidades (em quilos por dia) de diversos grãos eletrofundidos (linhas da tabela) que podem ser produzidos por
10 processos (conjuntos de peneiras combinados com a regulagem de moinhos e britadores) diferentes (colunas de 1 a 10). Por exemplo, o conjunto 1 é capaz de produzir 2000 quilos do produto EC31_36, 600 quilos do produto EC31_120, 500 quilos do produto EC31_150, e assim por diante, totalizando 27700 quilos por dia (última linha da tabela). Note que um mesmo produto pode ser produzido por diferentes conjuntos de peneiras e em quantidades diferentes, sendo que, uma vez montada a linha de produção e escolhido um conjunto de peneiras para operar, alguns produtos produzidos por este conjunto podem não ter sido demandados. Isso acontece porque no processo escolhido já estão definidos os produtos e as quantidades de cada produto a serem produzidos no dia, então, é comum carregar estoques de produtos ainda sem demanda por longos períodos e até mesmo ao final do horizonte planejado. Uma forma de diminuir a quantidade carregada é utilizar o excesso dos produtos de grãos médios e grossos como matéria-prima de grãos finos que tenham demandas. Isso implica custos adicionais, uma vez que se torna necessário retornar esses grãos ao moinho para que sejam triturados mais uma vez.

Atualmente, a programação da produção, feita manualmente pelo PCP da empresa, prioriza prazos de entrega e clientes, devido aos prazos de entrega, em geral apertados (negociados pelo departamento de vendas), e ao nível de serviço aos clientes preferenciais. Clientes também estão tendendo a dividir pedidos mensais em pedidos semanais ou quinzenais, o que resulta num maior número de pedidos com pequenas quantidades demandadas. Em casos em que não seja possível programar a produção de alguns itens de forma a atender completamente a suas demandas num certo período, a empresa renegocia os prazos de entrega com os clientes de forma a minimizar seu descumprimento. Portanto, uma melhor programação da produção reduz o número de renegociações de prazos de entrega entre a empresa e seus clientes. Além disso, também facilita a aplicação de certas técnicas de programação, como o uso de "horizonte rolante" (Clark e Clark, 2000).

\section{Modelagem do problema}

A modelagem do problema aqui estudado baseia-se numa combinação dos problemas de seleção de processos e dimensionamento de lotes, definidos a seguir.

\subsection{Problema de seleção de processos}

Neste tipo de problema as demandas dos produtos são fixadas ao longo de um horizonte de planejamento. Cada produto pode ser produzido por diferentes processos alternativos. Os custos de produção e os recursos utilizados dependem do processo escolhido. Os recursos têm limites de disponibilidade no período e vários produtos competem por estes recursos, de acordo com o processo de 
Tabela 1. Quantidades (em quilos por dia) de grãos eletrofundidos (linhas da tabela) produzidos pelos processos 1-10 de peneiras (colunas).

\begin{tabular}{|c|c|c|c|c|c|c|c|c|c|c|}
\hline & 1 & 2 & 3 & 4 & 5 & 6 & 7 & 8 & 9 & 10 \\
\hline EC31_36 & 2000 & 2000 & 2000 & 2000 & 2000 & 2000 & 2000 & 2000 & 2000 & \\
\hline EC31_120 & 600 & & 600 & & 600 & & 1000 & & 600 & \\
\hline EC31_150 & 500 & & & 300 & 300 & & & 300 & 300 & \\
\hline EC31_180 & 300 & & 300 & & 300 & & 500 & & & \\
\hline EC31_220 & 300 & & & 300 & & & & & & \\
\hline EG52_120 & & 500 & & 500 & & 500 & & 500 & & 10000 \\
\hline EG52_150 & & 300 & 300 & & & 200 & 500 & & 800 & \\
\hline EG52_180 & & 300 & & 300 & & & & 300 & 1000 & \\
\hline EC31R_3-8_1-4 & 2000 & 2000 & 2000 & 2000 & 1000 & 2000 & 2000 & 2000 & 3600 & 10000 \\
\hline EC31R_3-5_7 & 5000 & 5000 & 5000 & 5000 & 4000 & 5000 & 5000 & 5000 & 4800 & \\
\hline EC31R_5-16_4 & 2000 & 2000 & 2000 & 2000 & 1000 & 2000 & 2000 & 2000 & 5000 & 5000 \\
\hline EC31R_08_F & 5000 & 5000 & 10000 & 8000 & 7000 & 7000 & 10000 & 10000 & & \\
\hline EC31R_08_F1 & 6000 & & & & 800 & & 1500 & 1000 & & \\
\hline EC31R_08_F2 & & 400 & & 500 & & 1000 & & 1000 & & \\
\hline EC31R_08_F3 & & & & & 500 & & 1000 & 1000 & & 2000 \\
\hline EC31R_08_F4 & 2000 & & 2000 & & & 1000 & & & & \\
\hline EC31R_08_F5 & & 900 & & & & & 1000 & & & \\
\hline EC31R_08_G & & & 1000 & 1000 & 1000 & & & & & \\
\hline EC31R_08_G1 & & 1000 & 1000 & & 1000 & & & & & \\
\hline EC31R_08_G2 & & & & & & 1000 & & 1000 & 8000 & \\
\hline EC31R_08_G3 & 2000 & & & 5000 & & 4000 & & & & \\
\hline EC31R_08_G4 & & 8000 & & & 7000 & & & & & \\
\hline EC31R_08_G5 & & & 1200 & 800 & 500 & & 1000 & & & \\
\hline Produção Conj. & 27700 & 27400 & 27400 & 27700 & 27000 & 25700 & 27500 & 26100 & 26100 & 27000 \\
\hline
\end{tabular}

produção escolhido. O problema consiste em determinar o quanto produzir de cada produto em cada processo, de maneira a minimizar os custos de produção, sujeito às restrições de limitação de recursos e atendimento da demanda (Jonhson e Montgomery, 1974; Hax e Candea, 1984; Williams, 1993; Graves et al., 1994, Nahmias, 1995).

\subsection{Problema de dimensionamento de lotes (lot-sizing)}

O problema de dimensionamento de lotes, de forma geral, consiste em planejar a quantidade de itens a ser produzida em cada período ao longo de um horizonte de tempo finito, de modo a atender à demanda e otimizar um critério, por exemplo, minimizar custos ou maximizar a contribuição ao lucro (Johnson e Montgomery, 1974; Hax e Candea, 1984; Askin e Standridge, 1993). Para Feng e Cheng (1998), resolver problemas de dimensionamento de lotes é cada vez mais difícil devido às técnicas industriais em geral ficarem cada vez mais complexas. Eles apontam cinco dimensões de complexidade para os problemas de dimensionamento de lotes monoestágio:

(i) disponibilidade limitada de múltiplos recursos;

(ii) existência de vários produtos compartilhando os mesmos recursos; (iii) demanda variável período a período, e vários períodos no horizonte de planejamento;

(iv) tempos de setup (ou preparação); e

(v) custos de setup para produzir um lote de um determinado produto. Quando considerados tempos e custos de setup, os problemas de dimensionamento de lotes tornam-se difíceis de resolver (NP-difíceis na teoria de complexidade), podendo ser formulados como problemas de programação linear inteira mista com variáveis de decisão, indicando a produção, ou não, de cada produto em cada período. Em Bitran e Yanasse (1982), é discutida a complexidade computacional de problemas de dimensionamento de lotes.

Diversos estudos são encontrados na literatura com modelos e métodos de solução para cada tipo de problema. Por exemplo, para o problema de dimensionamento de lotes capacitado - capacited lot-sizing problem (CLSP) (Both et al., 1984; Gunnther, 1987; Trigeiro et al., 1989; Maes et al., 1991), o problema discreto de dimensionamento e programação de lotes - Discrete Lotsizing and Scheduling Problem (DLSP) (Fleischmann, 1990; Salomon, 1991; Salomon et al., 1997; Eijl e Hoesel, 1997; Bruggemann e Jahnke, 1999) e o problema de 
dimensionamento de lotes com setup contínuo - Continuous Setup Lot-sizing Problem (CSLP) (Bitran e Matsuo, 1986; Karmarkar et al., 1987; Matta e Guignard, 1989; Kimms, 1996). Drexl e Kimms (1997) e Karimi et al. (2003) apresentam revisões dos diversos problemas de dimensionamento de lotes, incluindo o problema geral de dimensionamento e programação de lotes - General Lot-sizing and Scheduling Problem (GLSP). Outros estudos recentes com problemas de dimensionamento de lotes são encontrados em Armentano et al. (1999), Clark e Clark (2000), Haase e Kimms (2000), Staggemeier e Clark (2001), Ferreira (2002), Araujo (2003), Toso (2004) e Fleszar e Hindi (2004).

\subsection{0 problema da separação de grãos na empresa}

No presente estudo de caso, a separação dos grãos é feita por um conjunto de peneiras. A programação da produção dos grãos não está apenas restrita à escolha das peneiras que são utilizadas para formar o conjunto, mas também depende da programação dos moinhos. Pela regulagem (aperto) dos moinhos define-se uma maior quantidade de grãos finos ou grossos (existem várias curvas de produção com diferentes distribuições granulométricas possíveis de implementar; Pileggi et al., 1998). Assim, cada processo consiste basicamente na regulagem dos moinhos e na escolha de um conjunto de peneiras para utilização num dia de trabalho. Na Figura 2 são apresentados dois gráficos de curvas de produção de grãos nos moinhos com diferentes distribuições granulométricas. $\mathrm{O}$ gráfico da esquerda, por exemplo, apresenta uma curva que produz maiores quantidades de grãos grossos (a curva é mais acentuada à esquerda do gráfico). Já o gráfico da direita representa uma curva de produção de grãos finos.

O tamanho do lote do processo pode variar em cada período (dia de trabalho). Se os grãos estão em uma curva mais grossa, a produção em toneladas é maior. Por outro lado, quanto mais fina for a curva, menor será o montante produzido. O conjunto de peneiras define quais os produtos selecionados (produzidos), enquanto a regulagem dos

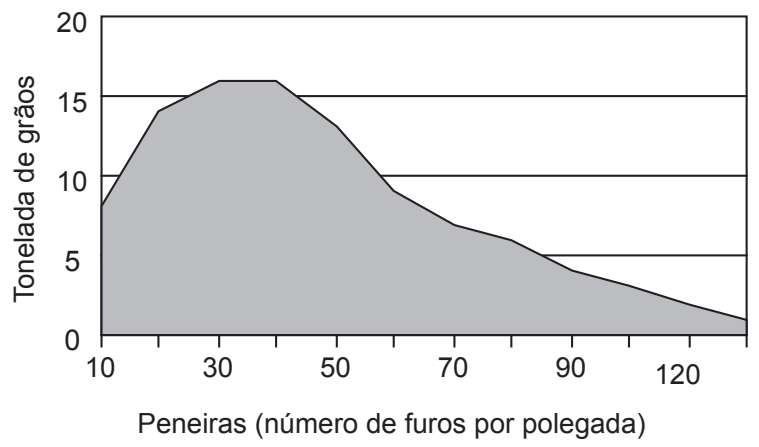

Figura 2. Curvas de produção de grãos nos moinhos. moinhos define a quantidade de cada um destes produtos. Em cada dia de trabalho, a empresa deseja realizar no máximo um setup de processo; isso porque os tempos de setup das máquinas são muito altos. Em outras palavras, a empresa deseja evitar a troca de processo ao longo do dia, uma vez que parte da preparação do processo é feita antes de iniciar o primeiro turno de trabalho (é importante salientar que os turnos não totalizam 24 horas por dia). O mix de produtos a ser produzido num dia é definido no processo, e há diversas restrições tecnológicas para a definição do processo. Para cada período é necessário encontrar o processo que melhor atenda à quantidade demandada de cada produto, sabendo-se que, por um lado, dificilmente todos os produtos demandados serão produzidos por este processo, por outro lado, provavelmente outros produtos não demandados serão produzidos. A programação da produção de grãos ainda está condicionada a:

a. O horizonte de programação adotado em geral é de um mês, o que se resume normalmente a 19 períodos, que são os dias em que há produção na empresa;

b. Novos pedidos de clientes podem ser aceitos ao longo de um horizonte já programado. Ou seja, dentro de uma programação definida, pode ser necessário refazer a programação para satisfazer à nova demanda (isto pode ocorrer inclusive nos períodos "congelados" de um horizonte rolante);

c. Não se considera o estoque inicial dos produtos; o que seria estoque é debitado da quantidade demandada destes produtos nos primeiros períodos, antes de se fazer a programação; e

d. Considera-se que os produtos sejam produzidos em monoestágio (demanda independente), portanto não existe ordem de precedência na produção de itens.

Nesse problema, as restrições de capacidade das máquinas são consideradas no momento da elaboração dos processos de produção, ou seja, cada processo de produção leva em conta as restrições de capacidade dos equipamentos da linha de produção.

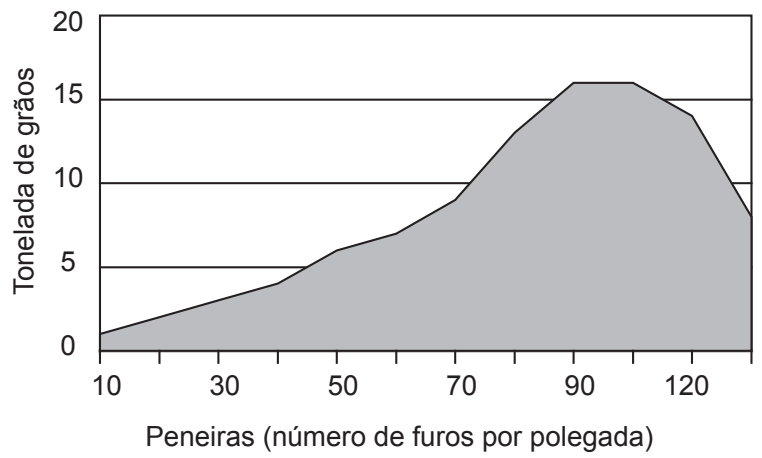




\subsection{Modelagem do problema da empresa}

Conforme mencionado, os modelos apresentados aqui, são uma combinação de modelos da literatura para problemas de seleção de processos e dimensionamento de lotes. O PCP da empresa tem dificuldades para estimar com precisão custos diários de estocagem e falta de produtos; também tem dificuldades para gerar programas de produção factíveis devido aos prazos de entrega em geral apertados (negociados pelo departamento de vendas), e ao crescimento de pedidos semanais e quinzenais (com quantidades demandadas menores) em relação aos pedidos mensais. Assim, em função destas dificuldades e do tempo de setup para mudança de processo ser muito grande, por simplicidade admite-se inicialmente que o problema consista em encontrar um programa de produção que, no menor número possível de períodos, seja capaz de atender a toda demanda, utilizando no máximo um processo por período (ou seja, que não necessite trocar de processo ao longo do dia).

Primeira proposta - Minimizar o Número de Períodos (MNP) necessários para a produção dos itens demandados: a idéia é produzir o quanto antes, ou seja, mesmo sendo possível postergar a produção num certo período, isto não é feito devido à oportunidade de receber novos pedidos e ainda poder produzi-los durante o horizonte de planejamento. Isto pode acarretar em carregar maiores estoques devido à possibilidade de se estar adiantando a produção de alguns itens, por outro lado, maximiza a produtividade da planta.

$$
\begin{array}{cl}
\text { Modelo } 1 \text { - } & \text { MNP: } \\
\text { variáveis: } & x_{j t} \text { : indica se o processo } j(j=1,2, \ldots, n) \\
& \text { é utilizado no período } t(t=1,2, . ., T) ; \\
\text { parâmetros: } & a_{i j} \text { : quantidade do item } i(i=1,2, \ldots, m) \\
& \text { produzido pelo processo } j \\
& d_{i t} \text { : demanda do item } i \text { no período } t . \\
\text { Min } \quad \mathrm{Z}=\sum_{t=1}^{T} \sum_{j=1}^{n} t x_{j t}
\end{array}
$$

Sujeito a $\sum_{t^{\prime}=1}^{T} \sum_{j=1}^{n} a_{i j} x_{j t^{\prime}} \geq \sum_{t^{\prime}=1}^{t} d_{i t^{\prime}} \quad i=1, . ., m \quad t=1, . ., T$ $\sum_{j=1}^{n} x_{j t} \geq 1$

$$
t=1, . ., T
$$

$$
\text { com } \quad x_{j t} \in\{0,1\} \text {, }
$$$$
j=1, . ., n, \quad t=1, . ., T
$$

A função objetivo (1) minimiza o número de períodos necessários para produção da demanda. A restrição (2) garante que a demanda de cada item $i$ em cada período $t$ seja atendida (a quantidade total produzida de um item até um certo período deve ser maior ou igual à demanda acumulada até este período). A restrição (3) impõe que no máximo um processo seja utilizado em cada período $t$. Note que o modelo acima pode ser visto como um modelo de dimensionamento de lotes que, em vez de "lotes de produtos", utiliza "lotes de processos" que produzem um conjunto de produtos. $\mathrm{O}$ modelo 1 também pode ser reescrito para que o valor da função objetivo $z$ corresponda literalmente ao número de períodos necessários (e de uma função de penalização), ou seja:

Min

$$
\mathrm{z}=\sum_{t=1}^{T} \sum_{j=1}^{n} x_{j t}
$$

Sujeito a restrições (2), (3), (4)

$$
\sum_{j=1}^{n} x_{j t} \geq \sum_{j=1}^{n} x_{j, t+1} \quad t=1, . ., T-1 .
$$

A restrição (6) impõe uma ordem na atribuição das variáveis $x_{j t}$, de maneira a evitar que um período $t+1$ seja utilizado sem que o período $t$ também seja. Desta forma a solução sempre utiliza apenas os primeiros períodos disponíveis para produção. Os resultados são os mesmos do modelo 1.

Segunda Proposta - Minimizar a Falta de Produção (MFP): como a restrição de demanda (2) freqüentemente é infactível na prática (devido a prazos de entrega muito apertados negociados pelo departamento de vendas), o modelo 2 a seguir utiliza variáveis de folga (excesso) e de falta na restrição (2) para garantir factibilidade.

Modelo 2 - MFP:

Variáveis adicionais: $f_{i t}$ : falta do item $i$ no período $t$; $e_{i t}:$ folga (excesso) do item $i$ no período $t$.

Min

$\mathrm{z}=\sum_{\mathrm{t}=1}^{T} \sum_{i=1}^{m} f_{i t}$

Sujeito a:

$\left(\sum_{t^{\prime}=1}^{T} \sum_{j=1}^{n} a_{i j} x_{j t^{\prime}}\right)+f_{i t}-e_{i t}=\sum_{t^{\prime}=1}^{t} d_{i t^{\prime}} \quad i=1, . ., m, \quad t=1, . ., T$

$\sum_{j=1}^{n} x_{j t} \leq 1$

$t=1, . ., T$

com

$x_{j t} \in\{0,1\}$, fit $\geq 0$, eit $\geq 0,=1, . ., n$,

$$
i=1, . ., m, t=1, . ., T
$$

A função objetivo (7) minimiza a falta de produção dos itens demandados. A restrição (8) de demanda agora inclui as variáveis de folga e falta. Note que uma solução ótima do modelo 2 sempre satisfaz $f_{i t} e_{i t}=0$. Nos casos em que esta solução resulta em $z=0$, ou seja, todos os prazos de entrega são atendidos ao longo do horizonte programado, o modelo 2 também pode (assim como o modelo 1) reduzir o número de períodos necessários para a produção da demanda. Uma forma de se fazer isso é simplesmente redefinir a função objetivo (7) por: 


$$
\text { Min } \quad \mathrm{z}=K \sum_{t=1}^{T} \sum_{i=1}^{m} f_{i t}+\sum_{t=1}^{T} \sum_{j=1}^{n} t x_{j t}
$$

em que $K$ é um número suficientemente grande de maneira que o primeiro objetivo (minimizar a falta) domine o segundo (minimizar o número de períodos de produção).

Relaxação de Programação Linear dos modelos MNP e MFP.

A solução relaxada dos modelos 1 e 2 (com $\left(x_{j t} \in\{0,1\} \rightarrow 0 \leq x_{j t} \leq 1\right)$ fornece a proporção de tempo em que cada processo $j$ é processado em cada período $t$ (sem considerar o custo/tempo de setup). A solução relaxada pode ser útil para sugerir a geração de novos processos mais convenientes, do ponto de vista da demanda no horizonte de planejamento, por meio da combinação de processos existentes. Conforme discussão na seção 4.1.1, este é um tópico para pesquisa futura.

\subsection{Modelos MNP e MFP com tempos de setup}

Conforme já mencionado, a empresa não utiliza programas de produção com mais de um processo (setup) por período. Para investigar possíveis vantagens de se utilizar mais de um processo por período, os modelos 1 e 2 também foram estendidos para considerar explicitamente tempos de setup dentro do período.

\section{Modelo 3 - MNP com tempos de setup:}

Parâmetros adicionais: st: tempo de setup do processo $j$ (fração de um dia $0 \leq t_{j} \leq 1$ );

Variáveis adicionais: $q_{j t}$ :tempoutilizandooprocesso $j$ no período $t$ (fração de um dia: $\left.0 \leq q_{j t} \leq 1\right)$.

Min $\mathrm{z}=\sum_{t=1}^{T} \sum_{j=1}^{n} t x_{j t}$

Sujeito a:

$\sum_{t^{\prime}=1}^{t} \sum_{j=1}^{n} a_{i j} q_{j t^{\prime}} \geq \sum_{t^{\prime}=1}^{t} d_{i t^{\prime}}, \quad i=1, . ., m, t=1, \ldots, T$

$\sum_{j=1}^{n}\left(s t_{j} x_{j t}+q_{j t}\right) \leq 1$ $t=1, . ., T$

$q_{j t} \leq x_{j t}$,

$j=1, \ldots, n, t=1, . ., T$

com $x_{j t} \in\{0,1\}, q_{j t} \geq 0$,

$j=1, . ., n, t=1, \ldots, T$

A função objetivo (12) minimiza o número de períodos necessários para produzir a demanda. A restrição (13) garante que a demanda de cada item $i$ em cada período $t$ seja satisfeita. A restrição (14) garante que a soma dos tempos de setup e processamento dos processos do período $t$ deve ser menor ou igual a capacidade deste período (igual a 1 dia), e a restrição (15) impõe que o tempo de processamento do processo $j$ no período $t$ não deve ser superior à capacidade do período $t$. Este problema é difícil de ser resolvido. Note que o modelo 3 permite que mais de um processo seja utilizado por período, diferente dos modelos 1 e 2 . O mesmo pode ser feito para se obter o modelo correspondente ao modelo 2 (MFP) com tempos de setup:

\section{Modelo 4-CLSP: MFP com tempos de setup}

Min

$\mathrm{z}=\sum_{t=1}^{T} \sum_{i=1}^{m} f_{i t}$

Sujeito a:

$\left(\sum_{t^{\prime}=1}^{t} \sum_{j=1}^{n} a_{i j} q_{j t^{\prime}}\right)+f_{i t}-e_{i t}=\sum_{t^{\prime}=1}^{t} d_{i t}, \quad i=1, . ., M, t=1, \ldots, T$

$\sum_{j=1}^{n}\left(s t_{j} x_{j t}+q_{j t}\right) \leq 1$,

$t=1, . ., T$

$q_{\mathrm{jt}} \leq x_{\mathrm{jt}}$,

$$
j=1, . ., n, t=1, . ., T
$$

com

$$
\begin{aligned}
x_{j t} \in\{0,1\}, q_{j t} \geq 0, f_{i t} \geq, \quad j=1, . ., n, & i=1, . ., m,(21) \\
t & =1, \ldots, T
\end{aligned}
$$

Similarmente à discussão anterior, nos casos em que a solução do modelo 4 resulta em $z=0$, ou seja, não há falta de produção em qualquer período do horizonte programado, o modelo 4 também pode (assim como o modelo 1) reduzir o número de períodos necessários para a produção da demanda. Uma forma de se fazer isso é simplesmente redefinir a função objetivo (17) para:

$$
\text { Min } \quad \mathrm{z}=K \sum_{t=1}^{T} \sum_{i=1}^{m} f_{i t}+\sum_{t=1}^{T} \sum_{j=1}^{n} t x_{j t}
$$

Luche (2003) mostra que os modelos acima ainda podem ser estendidos para tratar problemas combinando decisões de dimensionamento e programação (seqüenciamento) de lotes de processos, tais como o DLSP, o PLSP (Proportional Lot-sizing and Scheduling Problem) e o GLSP. Outros objetivos da empresa tais como: minimizar o estoque carregado de produtos entre períodos, minimizar custos de produção, estocagem, pedidos pendentes, vendas perdidas, priorizar produtos pela contribuição ao lucro, priorizar clientes ou pedidos, entre outros, podem ser tratados por meio de adaptações nos modelos anteriores e/ou aplicando programação de metas (goal programming) (Ravidran et al., 1987). Obviamente a aplicação desta técnica envolve certas dificuldades, uma vez que os modelos contêm variáveis inteiras.

\section{Resultados computacionais}

Nos experimentos realizados foi utilizado um microcomputador com processador Pentium-II $450 \mathrm{MHZ}$ com $128 \mathrm{MB}$ de memória $R A M$ e sistema operacional Windows $2000^{\circledR}$. Para resolver os modelos matemáticos, foi utilizada a linguagem de modelagem GAMS $2.0 \mathrm{com}$ o solver CPLEX 7.0 (Brooke et al., 1992). Linguagens de modelagem, além de traduzirem os modelos para um solucionador (solver) de problemas de programação mate- 
mática, também são importantes ferramentas para reduzir o tempo despendido por especialistas na implementação de um modelo. Revisões de linguagens de modelagem são encontradas em Kao (1998) e Kiup (1993).

\subsection{Dados simulados}

Inicialmente foi criada uma lista fictícia com $n=10$ processos (inspirados nos processos reais da empresa) para produzir $m=15$ itens num horizonte de programação de $T=10$ períodos, com demanda especificada por período. Esta situação simulada, de menor escala que a situação real, permite uma análise mais simplificada da funcionalidade e consistência dos modelos e soluções. Os dados de entrada são apresentados abaixo nas Tabelas 2 e 3. Os detalhes dos modelos GAMS podem ser encontrados em Luche (2003). Os tempos computacionais para resolver tais modelos com os dados das Tabelas 2 e 3 foram pequenos, entre 1 e 10 segundos.

\subsubsection{Modelo MNP}

Usando os dados das Tabelas 2 e 3, a solução do modelo MNP (modelo 1) gerou um programa de produção com 8 períodos, dois a menos que os $T=10$ períodos disponíveis no horizonte de planejamento. Este programa satisfaz todas as demandas de todos os produtos nos períodos em que foram requisitados (Tabelas 4 e 5), mas resulta num estoque total de produtos no final do horizonte de programação de $26.100 \mathrm{~kg}$. Isso se deve ao fato de que cada processo utilizado produz uma quantidade já determinada de certos produtos. Produzir a demanda no menor número de períodos possível não garante o menor volume de estoque. Assim, é possível que existam outras

Tabela 2. Quantidade $\left(a_{i j}\right)$ em quilos produzidos por dia do item $i$ utilizando o processo $j$.

\begin{tabular}{lrrrrrrrrrr}
\hline & $\mathbf{1}$ & \multicolumn{1}{c}{$\mathbf{2}$} & \multicolumn{1}{c}{$\mathbf{3}$} & $\mathbf{4}$ & $\mathbf{5}$ & $\mathbf{6}$ & $\mathbf{7}$ & $\mathbf{8}$ & $\mathbf{9}$ & $\mathbf{1 0}$ \\
\hline EK8A-16 & 0 & 1000 & 2000 & 0 & 500 & 0 & 0 & 0 & 1000 & 700 \\
EK8A_20 & 0 & 1000 & 500 & 0 & 500 & 0 & 0 & 0 & 1000 & 700 \\
EK8A_24 & 0 & 0 & 500 & 0 & 300 & 0 & 0 & 0 & 200 & 700 \\
EK8A_30 & 0 & 0 & 500 & 0 & 300 & 0 & 0 & 600 & 200 & 0 \\
EK8A_36 & 0 & 0 & 500 & 0 & 0 & 0 & 0 & 700 & 200 & 0 \\
EK8A_46 & 0 & 0 & 0 & 1500 & 0 & 0 & 0 & 700 & 200 & 0 \\
EK8A_54 & 0 & 0 & 0 & 800 & 0 & 0 & 0 & 700 & 200 & 0 \\
EK8A_60 & 1500 & 0 & 0 & 1500 & 2000 & 3000 & 3000 & 0 & 1000 & 0 \\
EK8A_80 & 1000 & 1500 & 500 & 1500 & 1500 & 2000 & 2500 & 0 & 1000 & 0 \\
EK8A_100 & 300 & 300 & 0 & 500 & 300 & 700 & 800 & 0 & 1000 & 0 \\
EK8A_120 & 300 & 300 & 0 & 500 & 300 & 700 & 800 & 500 & 1000 & 0 \\
EK8A_150 & 250 & 250 & 0 & 0 & 200 & 500 & 700 & 300 & 1000 & 0 \\
EK8A_180 & 250 & 250 & 0 & 500 & 200 & 500 & 700 & 300 & 500 & 0 \\
EK8A_220 & 250 & 250 & 0 & 400 & 200 & 500 & 700 & 500 & 400 & 0 \\
EK8A_FFF & 200 & 200 & 0 & 0 & 300 & 0 & 600 & 500 & 400 & 0 \\
\hline
\end{tabular}

Tabela 3. Quantidade $\left(d_{i t}\right)$ em quilos demandada do produto $i$ no período $t$.

\begin{tabular}{lrrrrrrrrrr}
\hline & $\mathbf{1}$ & $\mathbf{2}$ & $\mathbf{3}$ & $\mathbf{4}$ & $\mathbf{5}$ & $\mathbf{6}$ & $\mathbf{7}$ & $\mathbf{8}$ & $\mathbf{9}$ & \multicolumn{1}{c}{$\mathbf{1 0}$} \\
\hline EK8A-16 & 0 & 0 & 0 & 0 & 0 & 0 & 400 & 0 & 600 & 500 \\
EK8A_20 & 0 & 0 & 0 & 0 & 0 & 0 & 400 & 600 & 0 & 1000 \\
EK8A_24 & 0 & 0 & 1000 & 0 & 0 & 0 & 0 & 500 & 0 & 0 \\
EK8A_30 & 0 & 0 & 0 & 0 & 300 & 700 & 0 & 0 & 1000 & 0 \\
EK8A_36 & 0 & 0 & 0 & 0 & 300 & 0 & 0 & 1000 & 800 & 0 \\
EK8A_46 & 0 & 0 & 0 & 0 & 0 & 500 & 0 & 0 & 0 & 0 \\
EK8A_54 & 0 & 0 & 0 & 0 & 0 & 500 & 0 & 0 & 0 & 1500 \\
EK8A_60 & 0 & 0 & 0 & 1000 & 2000 & 0 & 0 & 0 & 1500 & 0 \\
EK8A_80 & 0 & 0 & 0 & 0 & 0 & 500 & 0 & 1000 & 0 & 2000 \\
EK8A_100 & 0 & 0 & 0 & 0 & 0 & 0 & 0 & 0 & 3000 & 0 \\
EK8A_120 & 0 & 0 & 0 & 0 & 0 & 0 & 0 & 0 & 0 & 1500 \\
EK8A_150 & 0 & 0 & 0 & 0 & 0 & 0 & 0 & 0 & 0 & 1500 \\
EK8A_180 & 0 & 0 & 0 & 0 & 0 & 0 & 0 & 0 & 0 & 1500 \\
EK8A_220 & 0 & 0 & 500 & 0 & 0 & 0 & 0 & 0 & 2000 & 0 \\
EK8A_FFF & 0 & 0 & 0 & 1000 & 0 & 0 & 0 & 0 & 0 & 2000 \\
\hline
\end{tabular}


combinações de processos produzindo menos estoque e utilizando o mesmo número de períodos.

Para tornar a análise mais interessante, foram mantidos os mesmos processos da Tabela 2, mas fez-se uma pequena mudança nos dados da Tabela 3 , acrescentandose um pedido de $1000 \mathrm{~kg}$ no período 3 para o produto EK8A_46. Por conveniência, a Tabela 6 reapresenta os dados da Tabela 3 com esta modificação, a qual torna impossível cumprir os prazos de entrega em cada um dos 10 períodos. Conforme esperado, o modelo MNP não é capaz de gerar uma solução viável; a infactibilidade ocorre devido à falta de capacidade para satisfazer a restrição de demanda (2) no período 3.

Tabela 4. Processos $j$ utilizados em cada período $t$ pelo modelo MNP (i.e., $\operatorname{com} x_{j t}=1$ ) para os dados das Tabelas 2 e 3.

\begin{tabular}{ccccccccc}
\hline Períodos & $\mathbf{1}$ & $\mathbf{2}$ & $\mathbf{3}$ & $\mathbf{4}$ & $\mathbf{5}$ & $\mathbf{6}$ & $\mathbf{7}$ & $\mathbf{8}$ \\
\hline Processos & 10 & 9 & 9 & 9 & 8 & 7 & 8 & 9 \\
\hline
\end{tabular}

Tabela 5. Excesso total de produção dos produtos gerado pela utilização dos processos da Tabela 4 referentes à Tabela 2, para a demanda da Tabela 3.

\begin{tabular}{lrcc}
\hline Produto & Estoque final & Produto & $\begin{array}{c}\text { Estoque } \\
\text { final }\end{array}$ \\
\hline EK8A-16 & 3200 & EK8A_80 & 3000 \\
EK8A_20 & 2700 & EK8A_100 & 1800 \\
EK8A_24 & 0 & EK8A_120 & 4300 \\
EK8A_30 & 0 & EK8A_150 & 3800 \\
EK8A_36 & 100 & EK8A_180 & 1800 \\
EK8A_46 & 1700 & EK8A_220 & 800 \\
EK8A_54 & 200 & EK8A_FFF & 200 \\
EK8A_60 & 2500 & \multicolumn{2}{c}{26100} \\
\hline \multicolumn{2}{c}{ Total de Excesso } &
\end{tabular}

Um experimento adicional foi relaxar as restrições de integralidade das variáveis inteiras $x_{\mathrm{jt}}$ do modelo MNP, permitindo utilizar mais de um processo por dia (sem levar em conta o tempo de setup adicional). A capacidade de produção de cada processo fica sujeita à proporção do tempo em que o processo é utilizado no dia. Utilizando os dados das Tabelas 2 e 6, o modelo 1 relaxado obtém uma solução factível, utilizando apenas 8 períodos para atender a toda demanda (com estoque total no final do horizonte programado de $17.520 \mathrm{~kg}$ ), mas com um total de 13 setups de processos, ou seja, alguns períodos envolvem até 3 setups. Soluções deste tipo não são utilizadas pela empresa devido aos grandes tempos de $s e$ tup envolvidos (que não estão considerados no modelo 1 relaxado). No entanto, esta solução relaxada pode ser útil para sugerir a geração de novos processos mais convenientes, do ponto de vista da demanda no horizonte de planejamento, por meio da combinação de processos existentes. Conforme mencionado, este é um tópico para pesquisa futura.

\subsubsection{Modelo MFP}

Aplicando o modelo MFP (modelo 2) para os dados das Tabelas 2 e 6, obtém-se a solução factível das Tabelas 7 e 8 , com todas as demandas satisfeitas até o final do horizonte programado. Note que no período 4 há falta de $100 \mathrm{~kg}$ do produto EK8A_FFF, que não aparece na Tabela 8 . Isso porque a quantidade devida de um produto em um período pode ser produzida em um próximo período antes do final do horizonte de planejamento. Assim, a falta do produto EK8A_FFF, que ocorreu no período 4, foi atendida no período 5 . O programa de produção utiliza os 10 períodos (Tabela 7) e resulta num estoque total no final do horizonte de $34.600 \mathrm{~kg}$ de produtos (Tabela 8).

Tabela 6. Quantidade $\left(d_{i i}\right)$ em quilos demandada do produto $i$ no período $t$ para a segunda simulação.

\begin{tabular}{lcrrrrrrrrr}
\hline & $\mathbf{1}$ & $\mathbf{2}$ & $\mathbf{3}$ & $\mathbf{4}$ & $\mathbf{5}$ & $\mathbf{6}$ & $\mathbf{7}$ & $\mathbf{8}$ & $\mathbf{9}$ & $\mathbf{1 0}$ \\
\hline EK8A-16 & 0 & 0 & 0 & 0 & 0 & 0 & 400 & 0 & 600 & 500 \\
EK8A_20 & 0 & 0 & 0 & 0 & 0 & 0 & 400 & 600 & 0 & 1000 \\
EK8A_24 & 0 & 0 & 1000 & 0 & 0 & 0 & 0 & 500 & 0 & 0 \\
EK8A_30 & 0 & 0 & 0 & 0 & 300 & 700 & 0 & 0 & 1000 & 0 \\
EK8A_36 & 0 & 0 & 0 & 0 & 300 & 0 & 0 & 1000 & 800 & 0 \\
EK8A_46 & 0 & 0 & 1000 & 0 & 0 & 500 & 0 & 0 & 0 & 0 \\
EK8A_54 & 0 & 0 & 0 & 0 & 0 & 500 & 0 & 0 & 0 & 1500 \\
EK8A_60 & 0 & 0 & 0 & 1000 & 2000 & 0 & 0 & 0 & 1500 & 0 \\
EK8A_80 & 0 & 0 & 0 & 0 & 0 & 500 & 0 & 1000 & 0 & 2000 \\
EK8A_100 & 0 & 0 & 0 & 0 & 0 & 0 & 0 & 0 & 3000 & 0 \\
EK8A_120 & 0 & 0 & 0 & 0 & 0 & 0 & 0 & 0 & 0 & 1500 \\
EK8A_150 & 0 & 0 & 0 & 0 & 0 & 0 & 0 & 0 & 0 & 1500 \\
EK8A_180 & 0 & 0 & 0 & 0 & 0 & 0 & 0 & 0 & 0 & 1500 \\
EK8A_220 & 0 & 0 & 500 & 0 & 0 & 0 & 0 & 0 & 2000 & 0 \\
EK8A_FFF & 0 & 0 & 0 & 1000 & 0 & 0 & 0 & 0 & 0 & 2000 \\
\hline
\end{tabular}




\subsubsection{Modelo MNP com tempos de setup}

Para enriquecer a análise da seção 4.1.1, foram incorporados no modelo MNP os tempos de setup dos processos (modelo 3). Devido à empresa não nos ter fornecido dados detalhados dos tempos de setup, por simplicidade, admitiu-se que todos os processos têm tempos de setup iguais a $10 \%$ de suas capacidades de produção. É importante lembrar que na Tabela 2, os valores de $a_{\mathrm{ij}}$ de cada processo consideram o tempo de setup do processo. Para o modelo 3 são utilizados os dados da Tabela 9, em que tais valores estão apresentados sem considerar os tempos de setup dos processos. A demanda continua a mesma da Tabela 6.

Tabela 7. Processos $j$ utilizados em cada período $t$ pelo modelo MFP (i.e., com $x_{j t}=1$ ) para os dados da Tabela 2 e Tabela 6.

\begin{tabular}{lcccccccccc}
\hline Períodos & $\mathbf{1}$ & $\mathbf{2}$ & $\mathbf{3}$ & $\mathbf{4}$ & $\mathbf{5}$ & $\mathbf{6}$ & $\mathbf{7}$ & $\mathbf{8}$ & $\mathbf{9}$ & $\mathbf{1 0}$ \\
\hline Processos & 4 & 10 & 5 & 7 & 8 & 9 & 8 & 3 & 9 & 7 \\
\hline
\end{tabular}

Tabela 8. Estoque ou falta total de produtos gerado pela utilização dos processos da Tabela 7 referentes à Tabela 2, para a demanda da Tabela 6.

\begin{tabular}{lclc}
\hline Produtos & Estoque final & Produtos & Estoque final \\
\hline EK8A-16 & 3700 & EK8A_80 & 7000 \\
EK8A_20 & 1700 & EK8A_100 & 1400 \\
EK8A_24 & 400 & EK8A_120 & 3900 \\
EK8A_30 & 400 & EK8A_150 & 2700 \\
EK8A_36 & 200 & EK8A_180 & 2200 \\
EK8A_46 & 1800 & EK8A_220 & 1300 \\
EK8A_54 & 600 & EK8A_FFF & 300 \\
EK8A_60 & 7000 & \multicolumn{2}{c}{34600} \\
\hline \multicolumn{2}{c}{ Total de Excesso } & \multicolumn{2}{c}{}
\end{tabular}

Ao contrário do modelo 1 da seção 4.1.1 (que não tem solução factível no horizonte de 10 períodos), o modelo 3 encontra uma solução satisfazendo toda demanda de cada período (Tabelas 10 e 11). Esta solução utiliza 12 setups de processo em 8 períodos (cerca de 1,1 dias de preparação), resultando num estoque total no final do horizonte de $17.641 \mathrm{~kg}$. Esta solução envolve menos trocas de processo do que a solução do modelo 1 relaxado (com 13 setups, conforme seção 4.1.1), mas resulta num estoque total um pouco maior (17.641 contra $17.520 \mathrm{~kg}$ ). Comparando-se esta solução com a do modelo 2 (com 10 setups de processo em 10 períodos e estoque total de $34.600 \mathrm{~kg}$ - Tabelas 7 e 8), nota-se que o aumento de setups de processo, além de permitir atender a toda demanda em cada período, também reduz significativamente o estoque final.

\subsubsection{Modelo MFP com tempos de setup}

Similarmente à seção 4.1.3, aqui a análise da seção 4.1.2 é revista considerando o modelo MFP com tempos de setup (modelo 4). Também são utilizados os dados da Tabelas 9 e 6 . A solução deste modelo envolve 15 setups de processos nos 10 períodos de programação (Tabe-

Tabela 10. Proporção do tempo $\left(q_{j i}\right)$ que é utilizado o processo $j$ no período $t$ pelo modelo MNP com tempos de setup para os dados das Tabelas 6 e 9.

\begin{tabular}{ccccccccc}
\hline $\begin{array}{c}\text { Proces- } \\
\text { Sos }\end{array}$ & \multicolumn{7}{c}{ Períodos } \\
\hline & 1 & 2 & 3 & 4 & 5 & 6 & 7 & 8 \\
5 & 0,268 & & & & & & & \\
7 & & & & & 0,909 & 0,078 & & 0,117 \\
8 & 0,378 & & 0,909 & & & 0,831 & & \\
9 & & & & 0,909 & & & 0,909 & 0,434 \\
10 & 0,263 & 0,909 & & & & & & \\
\hline
\end{tabular}

Tabela 9. Quantidade $\left(a_{i j}\right)$ em quilos produzidos por dia do item $i$ utilizando o processo $j$ sem tempos de setup.

\begin{tabular}{|c|c|c|c|c|c|c|c|c|c|c|}
\hline & 1 & 2 & 3 & 4 & 5 & 6 & 7 & 8 & 9 & 10 \\
\hline EK8A-16 & 0 & 1110 & 2220 & 0 & 555 & 0 & 0 & 0 & 1110 & 777 \\
\hline EK8A_20 & 0 & 1110 & 555 & 0 & 555 & 0 & 0 & 0 & 1110 & 777 \\
\hline EK8A_24 & 0 & 0 & 555 & 0 & 333 & 0 & 0 & 0 & 222 & 777 \\
\hline EK8A_30 & 0 & 0 & 555 & 0 & 333 & 0 & 0 & 666 & 222 & 0 \\
\hline EK8A_36 & 0 & 0 & 555 & 0 & 0 & 0 & 0 & 777 & 222 & 0 \\
\hline EK8A_46 & 0 & 0 & 0 & 1675 & 0 & 0 & 0 & 777 & 222 & 0 \\
\hline EK8A_54 & 0 & 0 & 0 & 888 & 0 & 0 & 0 & 777 & 222 & 0 \\
\hline EK8A_60 & 1675 & 0 & 0 & 1675 & 2220 & 3330 & 3330 & 0 & 1110 & 0 \\
\hline EK8A_80 & 1110 & 1675 & 555 & 1675 & 1675 & 2220 & 2775 & 0 & 1110 & 0 \\
\hline EK8A_100 & 333 & 333 & 0 & 555 & 333 & 777 & 888 & 0 & 1110 & 0 \\
\hline EK8A_120 & 333 & 333 & 0 & 555 & 333 & 777 & 888 & 555 & 1110 & 0 \\
\hline EK8A_150 & 277,5 & 277,5 & 0 & 0 & 222 & 555 & 777 & 333 & 1110 & 0 \\
\hline EK8A_180 & 277,5 & 277,5 & 0 & 555 & 222 & 555 & 777 & 333 & 555 & 0 \\
\hline EK8A_220 & 277,5 & 277,5 & 0 & 444 & 222 & 555 & 777 & 555 & 444 & 0 \\
\hline EK8A_FFF & 222 & 222 & 0 & 0 & 333 & 0 & 666 & 555 & 444 & 0 \\
\hline
\end{tabular}


la 12), o que equivale a 1,4 dias de preparação, resultando num estoque total no final do horizonte de $25.228 \mathrm{~kg}$ (Tabela 13). Note que a solução do modelo 2 utilizou menos trocas de processo (10 setups, conforme Tabela 7), mas resultou num estoque total bem maior $(34.600 \mathrm{~kg}$, conforme Tabela 8). Isto ilustra o trade-off entre o número de trocas de processos (devido aos tempos de setup) e o estoque total de produtos no final do horizonte programado.

\subsection{Dados reais}

Uma lista limitada de processos usados pela empresa foi tomada como base inicial para a programação da produção. O motivo deve-se ao gigantesco número de possíveis processos de fabricação que podem ser implementados na fábrica. Isto porque num processo existe a combinação da produção de diferentes quantidades de vários produtos. Considerando que a produção média diária seja de 28 toneladas, que existam cerca de 50 produtos diferentes, e que os produtos possam ser produzidos em quantidades a partir de $300 \mathrm{~kg}$ (sendo $5 \mathrm{~kg}$ a escala mínima de incremento), isso resultaria num número de processos muito grande, tornando os modelos intratáveis computacionalmente. Técnicas de geração de colunas (processos) poderiam ser aqui utilizadas, assim como na literatura de problemas de corte (Morabito e Arenales, 1992). No entanto, aqui há dificuldades adicionais para

Tabela 11. Estoque total de produtos gerado pela utilização dos processos da Tabela 10 referentes à Tabela 9, para a demanda da Tabela 6.

\begin{tabular}{lccc}
\hline Produto & Estoque final & Produto & Estoque final \\
\hline EK8A-16 & 2059 & EK8A_80 & 2512 \\
EK8A_20 & 1559 & EK8A_100 & 570 \\
EK8A_36 & 46 & EK8A_120 & 3245 \\
EK8A_46 & 646 & EK8A_150 & 2622 \\
EK8A_54 & 146 & EK8A_180 & 1372 \\
EK8A_60 & 2271 & EK8A_220 & 593 \\
\hline \multicolumn{2}{c}{ Total de Excesso } \\
\hline
\end{tabular}

se definir processos viáveis, em função das restrições dos diversos equipamentos envolvidos na linha de produção.

Assim, devido ao problema do grande número de processos (ordem de milhares), a empresa forneceu inicialmente apenas os 140 processos padrões mais utilizados (para alimentar os modelos). Sabe-se que com base somente nesses 140 processos é possível que os modelos não sejam capazes de produzir resultados melhores do que os programados pelo PCP da empresa, uma vez que o PCP da empresa tem maior flexibilidade na definição e escolha de processos. Por outro lado, essa primeira aproximação pode permitir aos programadores do PCP encontrar resultados mais rápidos e possivelmente melhores do que os obtidos sem o uso do computador. Além do que, novos processos definidos pelos programadores podem ir sendo adicionados à lista de processos do modelo, para se testar seu benefício.

Desta maneira, os dados de entrada consistem inicialmente de $n=140$ processos, com o tempo de setup já descontado da capacidade de produção de cada processo, $m=50$ produtos e $T=19$ períodos no horizonte de planejamento. A programação realizada pelo PCP da empresa neste horizonte resultou em uma falta total de $13.450 \mathrm{~kg}$, ou seja, não consegue atender a todos os prazos de entrega. Em geral, os programadores da empresa necessitam de muitas horas (às vezes até dias) para chegar a um programa de produção aceitável.

Tabela 13. Estoque total de produtos gerado pela utilização dos processos da Tabela 12 referentes à Tabela 9, para a demanda da Tabela 6.

\begin{tabular}{lccc}
\hline Produto & Estoque final & Produto & Estoque final \\
\hline EK8A-16 & 2437 & EK8A_60 & 6095 \\
EK8A_20 & 423 & EK8A_80 & 5805 \\
EK8A_24 & 753 & EK8A_120 & 2815 \\
EK8A_30 & 126 & EK8A_150 & 1600 \\
EK8A_36 & 289 & EK8A_180 & 1823 \\
EK8A_46 & 1385 & EK8A_220 & 1262 \\
EK8A_54 & 415 & & \\
\hline \multicolumn{2}{c}{ Total de Excesso } \\
\hline
\end{tabular}

Tabela 12. Proporção do tempo $\left(q_{j t}\right)$ que é utilizado o processo $j$ no período $t$ pelo modelo MFP com tempos de setup para os dados das Tabelas 9 e 6.

\begin{tabular}{|c|c|c|c|c|c|c|c|c|c|c|}
\hline Processos & & & & & & & & & & \\
\hline & 1 & 2 & 3 & 4 & 5 & 6 & 7 & 8 & 9 & 10 \\
\hline 3 & & & & & 0,909 & & & & & \\
\hline 4 & 0,597 & & & & & & & & & \\
\hline 6 & & 0,251 & & & & 0,165 & & & & \\
\hline 7 & 0,312 & 0,280 & & 0,909 & & & & & & \\
\hline 8 & & & & & & 0,744 & 0,909 & 0,717 & 0,900 & \\
\hline 9 & & & & & & & & 0,192 & & \\
\hline 10 & & 0,378 & 0,909 & & & & & & & 0,909 \\
\hline
\end{tabular}




\subsubsection{Modelo MNP utilizando 140 processos}

Ao aplicar o modelo MNP (modelo 1) utilizando apenas os 140 processos iniciais, não foi obtida uma solução factível. A demanda de alguns itens em determinados períodos é maior do que a quantidade acumulada disponível para entrega. A empresa deseja que a demanda de cada item seja cumprida totalmente, e não apenas parcialmente. Um experimento adicional foi relaxar as restrições de integralidade das variáveis inteiras $x_{j t}$ do modelo MNP; mesmo assim o modelo relaxado não obteve uma solução factível. Os tempos computacionais requeridos pelo GAMS/CPLEX nestes experimentos foram de menos de 1 minuto.

\subsubsection{Modelo MNP utilizando 159 processos}

Foram então adicionados outros 19 processos aos dados de entrada, que correspondem a processos utilizados na programação do PCP da empresa (lembre-se que esta programação não satisfaz todos os prazos de entrega). Ao se aplicar o modelo MNP utilizando os 159 processos, ele também não foi capaz de gerar uma solução factível. Relaxando-se as variáveis inteiras $x_{j t}$ do modelo, obtevese uma solução factível utilizando apenas 14 períodos de produção, mas contendo até 6 processos sendo executados num mesmo dia de produção.

\subsubsection{Modelo MFP utilizando 140 processos}

O modelo MFP (modelo 2) foi então aplicado aos dados com 140 processos, para minimizar a falta. $O$ resultado obtido (falta total de $42.370 \mathrm{~kg}$ ) fica distante do resultado da programação feita pelo PCP da empresa $(13.450 \mathrm{~kg})$. Isso acontece devido ao fato de que o modelo trabalha com possibilidade de escolha dentre apenas 140 processos, enquanto que os programadores do PCP têm grande flexibilidade para formular novos processos que atendam melhor à demanda. O tempo computacional consumido pelo GAMS/CPLEX para obter tal programação é bem aceitável para as decisões envolvidas: cerca de 1 minuto.

\subsubsection{Modelo MFP utilizando 159 processos}

Similarmente à seção 4.2.2, resolveu-se o modelo MFP adicionando-se aos dados de entrada os 19 processos utilizados pelo PCP da empresa. Desta maneira, espera-se que o modelo encontre, na pior das hipóteses, uma solução pelo menos tão boa quanto a utilizada pelo PCP. De fato, nesta segunda simulação obteve-se um resultado melhor do que o da empresa: falta total de $10.475 \mathrm{~kg}$ num tempo computacional de 3 minutos. Convém salientar que a diferença é significativa (redução de $22 \%$ da falta). Ou seja, os 19 processos adicionais incluídos aos 140 processos padrões são importantes para uma programação da produção com melhor atendimento dos prazos de entrega.

\subsection{Considerações finais a respeito dos modelos utílizados}

O modelo MNP é útil quando a programação da produção pode ser cumprida sem atraso da produção de qualquer item, porém não permite obter uma solução de mínima falta para o problema quando este não for factível do ponto de vista de atraso zero. Neste caso deve ser utilizado o modelo MFP. Os experimentos da seção 4.2 sugerem que, nas situações reais, a qualidade das soluções é muito sensível ao conjunto de processos (disponíveis nos dados de entrada do modelo). Apesar da empresa usar freqüentemente cerca de 140 processos padrões, este conjunto isolado pode não ser capaz de gerar bons programas de produção. É necessário que outros processos explorando os padrões da demanda em cada período sejam também considerados pelos modelos. Isto sugere a importância da interação dos tomadores de decisão na utilização dos modelos, testando o benefício da inclusão de novos processos em função da carteira de pedidos de cada horizonte de planejamento.

\section{Conclusões e perspectivas para pesquisa futura}

Neste trabalho propõe-se uma abordagem de solução para o problema de programação da produção de grãos eletrofundidos, combinando modelos de seleção de processos e dimensionamento de lotes monoestágio. Os modelos combinados podem ser vistos como modelos de dimensionamento de lotes que, em vez de lotes de produtos, utilizam lotes de processos produzindo um conjunto de produtos. Os resultados mostram que os modelos são capazes de gerar soluções melhores do que as utilizadas pela empresa. Devido aos tempos computacionais relativamente baixos (se consideradas as decisões envolvidas) usando o GAMS/CPLEX, tal abordagem permite que sejam feitas várias simulações de programação da produção (explorando diferentes cenários), o que fornece flexibilidade e eficácia aos tomadores de decisão. Além disso, a abordagem também facilita a aplicação de técnicas de horizonte rolante, e permite aos departamentos de produção e vendas da empresa analisar rapidamente a incorporação de novos pedidos dentro do horizonte de planejamento.

Como observado nos experimentos computacionais, para que os modelos encontrem bons programas de produção, além dos processos padrões é importante a inclusão de novos processos que levem em conta o mix da demanda em cada período do horizonte de planejamento. Uma perspectiva interessante para pesquisa futura é a aplicação de técnicas de geração de colunas (processos) na solução destes modelos, de maneira semelhante aos modelos da literatura de problemas de corte (Morabito e Arenales, 1992). No entanto, o procedimento de geração de um processo viável para a fábrica de grãos eletrofundidos envolve certas 
dificuldades de modelagem, devido às restrições técnicas dos diversos equipamentos da linha de produção.

Nesta linha de pesquisa, as soluções dos modelos relaxados talvez possam ser úteis para ajudar na geração de novos processos, dado que resultam em vários processos num único período. A combinação destes processos num único processo pode ser considerada para se definir um novo processo mais adequado para o período. Quanto ao uso dos modelos MNP e MFP com tempos de setup (modelos 3 e 4), está na nossa agenda de pesquisa realizar um outro estudo na empresa para analisar em profundidade as vantagens e desvantagens das soluções com mais de um setup por período (em relação às soluções com no máximo um setup por período), o que poderia justificar uma mudança na política das empresas deste setor de evitar trocas de processo dentro do período.

\section{Agradecimentos}

Os autores agradecem aos dois revisores anônimos e à Gisele C. F. Pileggi pelos úteis comentários e sugestões. Também agradecem à Empresa Alcoa-EMAS (atualmente do grupo Treibacher Schleifmittel Brasil), em particular a Luis A. Camilotti e Marcelo Suster, pelo apoio e colaboração com esta pesquisa.

\section{Referências Bibliográficas}

ALCOA. Produtos químicos. Disponível em: <http://www. alcoa.com.br/produtos/quimicos.asp $>$. Acesso em: 21 outubro 2002.

ALLEN, T. Particle size measurement. 4. ed. Londres: Chapman and Hall, 1990, 806 p.

ARAUJO, S. A. Modelos e métodos para o planejamento da produção aplicados no setor de fundições. 2003. Tese (doutorado em Matemática Computacional) - Intituto de Ciências Matemáticas e Computação, Universidade de São Paulo, São Carlos, 2003.

ARMENTANO, V. A.; FRANÇA, P. M.; TOLEDO, F. M. B. A network flow model for the capacitated lot-sizing problem. Omega International Journal of Management Science, v. 27, n. 2, p. 275-284, 1999.

ASKIN, R.; STANDRIDGE, C. Modeling and analysis of manufacturing systems. New York: Wiley \& Sons, 1993.

BITRAN, G. R.; MATSUO, H. Approximations for the single - product capacitated lot size problem. Operations Research, v. 34, n. 1, p. 63-74, 1986.

BITRAN, G. R.; YANASSE, H. H. Computational complexity of the capacitated lot size problem. Management Science, v. 28, n. 10, p. 1174-1186, 1982.

BODT, M. A.; GELDERS, L. F.; WASSENHOVE, L. N. Lot sizing under dynamic demand conditions: a review. Engineering Costs and Production Economics, v. 8, p. 165-187, 1984.

BROOKE, A.; KENDRICK, D.; MEERAUS, A. GAMS: a user's guide (release 2.25). San Francisco: The Scientific Press, 1992.

BRUGGEMANN, W.; JAHNKE, H. The discrete lot-sizing and scheduling problem: Complexity and modification for batch availability. European Journal of Operational Research, v. 124, n. 3, p. 511-528, 1999.
CLARK, A. R.; CLARK, S. J. Rolling-horizon lot-sizing when set-up times are sequence-dependent. International Journal of Production Research, v. 38, n. 10, p. 2287-2307, 2000.

DREXL, A.; KIMMS, A. Lot sizing and scheduling: survey and extensions. European Journal of Operational Research, v. 99, n. 2, p. 221-235, 1997.

EIJL, C. A., HOESEL, C. P. M. On the discrete lot-sizing and scheduling problem with Wagner-Whitin costs. Operations Research letters, v. 20, n. 1, p. 7-13, 1997.

FENG, H.; CHENG, H. Solving mixed integer programming production planning problems with setups by shadow price information. Computers and Operations Research, v. 25, n. 12, p. 1027-1042, 1998

FERREIRA, D. Um modelo de dimensionamento de lotes aplicado à indústria de bebidas. 2002. 111 p., Dissertação (Mestrado em Matemática Aplicada), IBILCE , UNESP, S. J. do Rio Preto, 2002.

FLEISCHMANN, B. The discrete lot-sizing and scheduling problem. European Journal of Operational Research, v. 44, n. 3, p. 337-348, 1990.

FLESZAR, K.; HINDI, K. S. Solving the resource-constrained project problem by a variable neighborhood scheduling search. European Journal of Operational Research, v. 155, n. 2, p. 402-413, 2004.

GRAVES, S. C.; RINNOOY KAN, A. H. G.; ZIPKIN, P. H. Logistics of production and inventory. Handbook in operations research and management science. Amsterdam : North-Holland, 1994.

GÜNTHER, H. O. Planning lot sizes and capacity requirements in a single-stage production system. European Journal of Operational Research, v. 31, n. 2, p. 223-231, 1987. 
HAASE, K.; KIMMS, A. Lot sizing and scheduling with sequence-dependent setup costs and times and efficient rescheduling opportunities. International Journal of Production Economics, v. 66, n. 2, p. 159-169, 2000.

HAX, A.; CANDEA, D. Production and inventory management. New Jersey: Prentice-Hall; Englewood Cliffs, 1984.

JOHNSON, L. A.; MONTGOMERY. Operations research in production planning, scheduling and inventory control. New York: Wiley, 1974.

KAO, C. Performance of several nonlinear programming software packages on microcomputers. Computers and Operations Research, v. 25, n. 10, p. 807-816, 1998.

KARIMI, B. ;GHOMI, S. M. T. F; WILSON, J. M. The capacitated lot sizing problem: a review of models and algorithms. Omega International Journal of Management Science, v. 31, n. 5, p. 365-378, 2003.

KARMARKAR, U. S.; KEKRE, S.; KEKRE, S. The deterministic lotsizing problem with startup and reservation costs. Operations Research, v. 35, n. 3, p. 389-398, 1987.

KIMMS, A. Multi-level, single-machine lot sizing and scheduling (with initial inventory). European Journal of Operational Research, v. 89, n. 1, p. 86-99, 1996.

KIUP, C. A. C. Algebraic languages for mathematical-programming. European Journal of Operational Research, v. 67, n. 1, p. 25-51, 1993.

LUCHE, J. R. D. Otimização na programação da produção de grãos eletrofundidos: Um estudo de caso. 2003. Dissertação (Mestrado em Engenharia de Produção), Programa de Pós-graduação em Engenharia de Produção. Universidade Federeal de São Carlos. São Carlos, 2003.

MAES, J.; McCLAIN, J. O.; van WASSENHOVE, L. N. Multilevel capacitated lot sizing complexity and LP-based heuristics. European Journal of Operational Research, v. 53, n. 2, p. 131-148, 1991.

MATTA, R.; GUIGNARD, M. Production scheduling with sequence independent changeover cost. Pennsylvania: The Wharton School; University of Pennsylvania, 1989.

MORABITO, R.; ARENALES, M. Um exame dos problemas de corte e empacotamento. Pesquisa Operacional, v. 12 , n. 1, p. 1-20, 1992.

NAHMIAS, S. Production and operations analysis. Local: Irwin, Homewood, IL, 1995.

PILEGGI, R. et al. Desenvolvimento e aplicação de um software que automatiza o processo de combinação de matérias-primas na obtenção de produtos cerâmicos. Cerâmica, v. 44, n. 289, p. 189-195, 1998.

RAVINDRAN, A.; PHILLIPS, D. T.; SOLBERG, J. J. Operations research: principles and practice. New York: Wiley, 1987.

SALOMON, M. Deterministic lotsizing models for production planning. Berlin: Springer, 1991. (Lecture Notes in Economics and Mathematical Systems vol. 355).

SALOMON, M. et al. Solving the discrete lotsizing and scheduling problem with sequence dependent set-up costs and set-up times using th Travelling Salesman Problem with time windows. European Journal of Operational Research, v. 100, n. 3, p. 494-513, 1997.

STAGGEMEIER, A. T., CLARK, A. R. A survey of lot-sizing and scheduling models. In: $33^{\circ}$ SIMPÓSIO BRASILEIRO DE PESQUISA OPERACIONAL, 2001.

TOSO, E. Otimização do problema integrado de dimensionamento de lotes e programação da produção: Estudo de caso na indústria de rações. 2004. Dissertação (Mestrado em Engenharia de Produção). Programa de Pós-graduação em Engenharia de Produção. Universidade Federal de São Carlos. São Carlos, 2004.

TRIGEIRO, W. W.; THOMAS, L. J.; Mc CLAIN J. O. Capacitated lot sizing with setup times. Management Science, v. 35, n. 3, p. 353-366. 1989.

WILLIAMS, P. Model building in mathematical programming. New York: Wiley \& Sons, 1993. 


\section{OPTIMIZATION IN THE PRODUCTION SCHEDULING OF ELECTROCAST GRAINS: A CASE STUDY}

\section{Abstract}

This paper proposes optimization models to support Production Planning and Control (PPC) decisions in the electrocast grain industry. A case study of a company in the state of São Paulo was conducted aiming to increase its productivity and improve its customer service from the standpoint of meeting delivery dates. To this end, mixed integer linear programming models were applied to aid in production scheduling decisions. The models combine known models of process selection and single-stage lot sizing. Optimizing production scheduling in the electrocast grain industry is not a simple task, especially in terms of the lengthy equipment setup times, the diversity of products and the delivery date constraints. The results indicate that the models can generate better solutions than those found by the company.

Keywords: production scheduling, lot-sizing, electrocast grains. 